\title{
Research Progress of the Clinical Application of Small Cell Lung Cancer Related Tumor Markers and Biochemical Indicators
}

\author{
Lei Wang, Jianwen Qin* \\ Respiratory Department, Tianjin Chest Hospital, Tianjin 300222, China \\ *Corresponding author: Jianwen Qin, simple0603@163.com
}

\begin{abstract}
With continual advances in medicine, more studies on early diagnosis, therapeutic evaluation, and prognosis of lung cancer have emerged in recent years, particularly in regard to small cell lung cancer (SCLC), which is a pathological type of lung cancer with the highest grade. In the era of medical treatment scarcity, early diagnosis of SCLC, evaluation of treatment effect, recurrence factors, and prognosis are important to treat patients with SCLC, prolong their survival, and improve their life quality. The research on the changes of serum tumor markers and biochemical indicators in the early diagnosis and treatment of SCLC has gradually become a hot topic. Therefore, this article reviews the research progress of serum tumor markers and biochemical indicators of SCLC.
\end{abstract}

Keywords: SCLC; Neuron specific enolase; Pro-gastrin-releasing peptide; Carcinoembryonic antigen; CYFRA 21-1; Ddimer; Lactate dehydrogenase; Neutrophil-to-lymphocyte ratio

Publication date: July 2021; Online publication: July 30, 2021

\section{Introduction}

Lung cancer is one of the most malignant tumors with the highest morbidity and mortality worldwide. Based on histology and the sensitivity level to chemoradiotherapy, it can be classified as non-small cell lung cancer (NSCLS) or small cell cancer (SCLC) with a morbidity of 75-80\% and 15-20\%, respectively. SCLC has been on an increasing trend in recent years, and due to its insidious onset, rapid progress, and high malignancy, most patients are usually diagnosed at an advanced stage, in which they do not have the chance for surgical treatment ${ }^{[1]}$. Therefore, SCLC is characterized by poor prognosis and curative ratio; the median survival is approximately 3 to 4 months if treatment is delayed. Therefore, early clinical diagnosis of SCLC is a positive factor that influences the treatment effect. Recently, in studies regarding the early diagnosis of SCLC, related tumor markers and biochemical indicators have become the topic of interest, where the testing of tumor markers from patients' blood or body fluid samples provides an advantage in diagnosing the cancer at an early stage along with its minimally invasive and easily-retrievable samples, thus providing a large research room for the early diagnosis and treatment of SCLC in addition to the improvement of the life quality and survival of patients.

SCLC most commonly occurs among male patients, generally around the age of 40 to 60, along with chronic smoking. This disease is sensitive to both radiotherapy and chemotherapy, and if active treatment measures such as conventional surgery, radiotherapy, and chemotherapy are provided when the symptoms manifest at the early stage, patients may have long-term survival ${ }^{[2]}$. The diagnosis, staging, treatment effect evaluation, and progress of SCLC are closely associated with neuron specific enolase (NSE), pro-gastrinreleasing peptide (Pro-GRP), CYFRA 21-1, squamous cell carcinoma antigen (SCC Ag), carcinoembryonic 
antigen (CEA), lactate dehydrogenase (LDH), and D-dimer in serum. The research progress of serum tumor makers and biochemical indicators of SCLC are reviewed.

\section{Neuron specific enolase (NSE)}

NSE is an isozyme of $\gamma$-dimer of enolase in glycolysis. As a tumor marker of neuroendocrine tumors, it specifically exists in neurons and neuroendocrine cells. With its highest activity in brain tissues, the serum NSE level could be a quantitative index of central nervous system injuries and a neuroendocrine tumor marker. However, it has low expression in healthy population. The cytoplasm of SCLC tumor cells contains neuron endocrine granules, characterized by neuroendocrine differentiation, so SCLC is a tumor with neuroendocrine properties that can secrete neuron specific enolase. An increased serum NSE concentration level was found in several patients with early-stage SCLC, thus demonstrating that NSE is a reliable indicator for the early diagnosis of SCLC, and it is considered as a tumor marker with high specificity for SCLC. During the treatment of patients with SCLC, their floating serum NSE levels may reflect condition changes. In regard to all these, NSE testing is able to provide evidence for early detection, diagnosis, and treatment of SCLC patients. In recent years, as the most sensitive tumor marker with neuroendocrine properties, NSE has caused widespread concern from experts at home and abroad. Zhaolin Guo and another researcher ${ }^{[3]}$ had reported the fasting serum NSE concentration levels of 96 patients with SCLC, 221 patients with pulmonary interstitial fibrosis, and 176 healthy participants from the control group by immunofluorescence. It was found that compared with the control group, the NSE concentration levels of patients with SCLC were higher, and there was a statistically significant difference $(p<0.01)$. In addition, the NSE levels of patients with pulmonary interstitial fibrosis were higher than those in the control group, without any statistically significant difference $(p>0.01)$. Yanjun Yin and other researchers [4] retrospectively analyzed the NSE concentration in 298 cases of SCLC and 368 cases of NSCLC and lung benign diseases. They demonstrated that the serum expression of NSE among SCLC patients was higher with significant differences to benign diseases, such as other pathological types of lung cancer, tuberculosis, and pneumonia. Without a significant increase of NSE in NSCLC patients, NSE can be used to detect and diagnose SCLC from NSCLC and the early screening of lung diseases. It also plays an important role in the early diagnosis and differential diagnosis of benign and malignant lung diseases. The research conducted by Pedrovic and other researchers ${ }^{[5]}$ indicated that the diagnosis sensitivity of SCLC may reach up to $80 \%$ with the specificity of $95 \%$ if the threshold of serum NSE is $19 \mathrm{ng} / \mathrm{ml}$. Several literatures also indicted that expression of NSE may be associated with the clinical staging of SCLC. Jianbo Zhou ${ }^{[6]}$ retrospectively analyzed 218 patients with SCLC and found that the serum NSE levels in extensive stage SCLC patients were much higher than those with limited stage SCLC $(32.5 \mathrm{ng} / \mathrm{ml}$ versus $14 \mathrm{ng} / \mathrm{ml}$, respectively; $p<0.001)$. Lifen Ren ${ }^{[7]}$ also confirmed that the NSE concentrations in stage III-IV SCLC patients were higher than those of stage I-II patients with statistically significant difference $(p<0.01)$. In addition, the NSE level was positively correlated with the number of metastases. Many studies have shown that the expression of serum NSE increases along with the clinical staging, which means that the NSE concentration in patients with middle and advanced stage is higher than that of early-stage patients while the NSE level in extensive stage is higher than that of the limited stage.

SCLC is a highly malignant tumor with strong invasiveness, prone for metastasis, and frequent recurrence after effective treatment. The 5-year survival rate of limited stage patients is approximately $25 \%$ ${ }^{[8]}$, and the median survival time of untreated patients is only 2 to 4 months ${ }^{[9]}$. It has been reported in a literature that the changes of NSE level in SCLC patients before and after chemotherapy are significantly related to the evaluation of the treatment effect ${ }^{[10]}$. Therefore, as a tumor marker, NSE is an important indicator to evaluate chemotherapy effect since patients who have responded to the treatment have an obvious decline in their serum NSE level ${ }^{[11]}$. Moreover, according to a study by Schneider and other 
researchers ${ }^{[12]}$, the serum NSE level of SCLC patients who have complete response (CR) or partial response (PR) after effective treatment reduced to the normal level $(p=0.001)$. In addition, the NSE in patients with stable disease (SD) had no significant change whereas the NSE levels in patients with progressive disease (PD) were raised. Another research showed that NSE could evaluate the survival of SCLC patients, where the survival of patients with NSE more than $19 \mathrm{ng} / \mathrm{ml}$ was significantly longer than that of patients with NSE less than $19 \mathrm{ng} / \mathrm{ml}(15 \text { months versus } 10 \text { months, respectively; } p<0.001)^{[5]}$. Using univariate Cox regression analysis, Weili Wang and other researchers ${ }^{[13]}$ demonstrated that NSE is associated with overall survival and the mortality risks of both NSE more than $48.9 \mathrm{ng} / \mathrm{mL}$ and NSE $32.6 \sim 48.9 \mathrm{ng} / \mathrm{mL}$ increased by 1.90 times and 1.57 times, respectively.

Certainly, there are many factors such as benign diseases or nerve system diseases which may lead to the increase of NSE. Furthermore, there may be false positive results due to hemolysis or external interference factors. Hence, for future research, we hope for more in-depth research on the influence of NSE on SCLC patients, including prediction and its evaluation significance. Discussions on its possible mechanisms are encouraged to provide new research perspectives as well as research evidence for the development of clinical treatment of SCLC patients with brain metastasis.

\section{Pro-gastrin-releasing peptide (pro-GRP)}

Pro-GRP is a precursor structure of gastrin-releasing peptide, existing in lung tissues of fetus, newborn, and patients with primary lung cancer, especially SCLC. Separated from non-gastric antrum tissues in pigs, GRP is a bombesin-like peptide, consisting of 27 amino acids. It is named GRP because it promotes gastrin secretion ${ }^{[14]}$. Research have found that SCLC can synthesize and release GRP, which stimulates tumor growth by auto- and para-secretion. It is also involved in the progress of tumor metastases. However, GRP is unstable in serum with only a half-life of 2 minutes; thus, it is not indicated in clinical tests. On the other hand, pro-GRP (31 98) is more stable in serum compared to the former ${ }^{[15]}$ and it is confirmed that its content in the serum is positively related to GRP ${ }^{[16]}$. Therefore, as a specific tumor marker SCLC, proGRP has showed its huge potential.

The serum pro-GRP of a normal person is commonly no more than $50 \mathrm{pg} / \mathrm{ml}$, and the upper limit of pro-GRP concentration in approximately $97 \%$ of patients with benign diseases is no more than $80 \mathrm{pg} / \mathrm{ml}^{[17]}$. Pawel and other researchers ${ }^{[18]}$ had tested 1750 blood samples with 667 cases of lung cancer and divided it into SCLC and NSCLC. The results showed that the expression of serum pro-GRP in SCLC patients was much higher than the healthy group, benign diseases group, and the NSCLC group, with statistically significant differences $(p<0.01)$. In addition, similar studies that had been performed by local researchers achieved the same results. Petrovic and other researchers ${ }^{[5]}$ reported that when serum pro-GRP is higher than $58 \mathrm{pg} / \mathrm{ml}$, the diagnosis sensitivity of SCLC is $84 \%$ while its specificity is $92 \%$; the meta-analysis conducted showed that the sensitivity and specificity were higher than another tumor marker - NSE. In another research, it was reported that pro-GRP can partially recognize the clinical staging of SCLC. Yiping $\mathrm{Li}$ and another researcher ${ }^{[19]}$ verified that the pro-GRP concentration in extensive stage SCLC patients is significantly higher than that of limited stage SCLC patients $(123 \mathrm{pg} / \mathrm{ml}$ versus $48.5 \mathrm{ng} / \mathrm{ml}$, respectively; $p$ $<0.001$ ). In addition, her article also suggested that pro-GRP has greater significance in the recognition of SCLC clinical staging compared to NSE.

Pro-GRP has recognition effect on the treatment of SCLC patients. Niho and other researchers [20] retrospectively analyzed 66 SCLC patients and found that $67 \%$ of patients' serum pro-GRP levels changed with their disease; that is, pro-GRP increased before chemotherapy, decreased after chemotherapy, and increased again with tumor recurrence. Jianbo Zhou ${ }^{[6]}$ also demonstrated that the pro-GRP levels among SCLC patients with different treatment effects have significant differences, of which CR group $<$ PR group $<\mathrm{SD}$ group $<$ PD group $(p<0.05)$. 
Pro-GRP is also an important and independent indicator to identify the prognosis of SCLC patients. Niho and other researchers ${ }^{[20]}$ reported that in 66 SCLC patients with tumor recurrence, the pro-GRP concentration in $83 \%$ of patients had abnormalities prior to the diagnosis of the recurrence. Okusaka and other researchers ${ }^{[21]}$ reported that with SCLC recurrence, the pro-GRP concentration in $86 \%$ of patients raised again and there was in an average of approximately 35 days in advance to the manifestation of clinical symptoms. This demonstrates that pro-GPR could work as a sensitive indicator for early prediction of the recurrence of SCLC and compared to NSE, there is a statistically significant difference. In a study by Shibamaya and other researchers ${ }^{[22]}$, the median survival time and 5-year survival rate of SCLC patients with high serum pro-GRP concentration were significantly lower than those of SCLC patients with low serum pro-GRP concentration (16.4 months versus 25.7 months; $10.6 \%$ versus $32.9 \%$; $p<0.05$ ).

Data have shown that false positive pro-GRP is mainly from patients with renal insufficiency. Other than that, pro-GRP concentration in patients with colorectal cancer, thyroid cancer, and prostate cancer may also increase ${ }^{[24]}$, but the total concentration is lower than SCLC patients $(P<0.05)$. Therefore, when clinical pro-GRP test is used to diagnose and estimate the treatment effect or recurrence for SCLC patients, false positives that occur due to renal insufficiency and other malignant tumors should be excluded.

\section{Carcinoembryonic antigen (CEA)}

CEA is an acid glycoprotein that is primarily extracted from colorectal cancer and fetal embryonic tissue, with the characteristic of human embryonic antigen, thus being entitled carcinoembryonic antigen. Numerous studies have found that CEA does not only increases in gastrointestinal cancer, but also in lung cancer, breast cancer, and other malignant cancers ${ }^{[25-28]}$. In a study by Siwei Yang and other researchers ${ }^{[29]}$, the comprehensive levels of combined detection of NSE and CEA for patients with SCLC were higher than those in lung cancer and squamous cell lung cancer. Therefore, CEA is valuable to SCLC diagnosis, and the combination of other tumor markers can improve the detection rate of SCLC.

\section{CYFRA 21-1}

Cytokeratin is a subunit that forms one of the structural proteins of epithelial cells - intermediate filament. Cytokeratin-19 (CYK-19) is a type-I keratin (acidic protein) and it is the smallest member in the keratin family. CYK-19 is widely distributed in stratified or squamous epithelium. In malignant epithelial cells, activated protease can accelerate cell degeneration, releasing a large amount of cytokeratin segments into the blood. Its soluble segments could specifically combine with two monoclonal antibodies - Ks19.1 and BM19.21, thus being called CYFRA 21-1. CYFRA 21-1 exists in malignant lung cancer tissues, and has full content, especially a high expression in squamous cell lung cancer ${ }^{[30]}$.

CYFRA 21-1 is a tumor marker used for lung cancer detection, particularly having a significant value in diagnosing NSCLC ${ }^{[31]}$. The positive detection rates are known to be $75 \%$ to $85 \%$ in regard to NSCLC. The serum concentration of CYFRA $21-1$ is positively correlated with the clinical staging of tumors. It is also an effective indicator for the evaluation of early recurrence for lung cancer surgery as well as the effects before and after radiotherapy and chemotherapy ${ }^{[32]}$. The diagnosis sensitivities of CYFRA 21-1 to the pathological patterns of lung cancer are successively such that squamous carcinoma $>$ adenocarcinoma $>$ large cell carcinoma > small cell carcinoma ${ }^{[33]}$. Besides that, CYFRA 21-1 has a certain positive rate to other tumors, including invasive bladder cancer, head and neck cancer, breast cancer, cervix cancer, and gastrointestinal cancer ${ }^{[34-37]}$. Shufang Qin and another researcher had selected 100 patients who were newly diagnosed with SCLC and found that the concentration of CYFRA 21-1 among patients in the SCLC group was significantly higher than those of the benign disease group and healthy control group, with a statistically significant difference $(p<0.05)$. In addition to that, the higher the clinical staging, the concentration of CYFRA 21-1 among SCLC patients was significantly higher. The difference among the four groups was 
statistically significant $(p<0.05)$ and the maximum specificity of three combined detection was $100 \%{ }^{[38]}$.

In general, CYFRA 21-1 has more diagnostic value to NSCLC, whereas combined detection is more suitable for SCLC.

\section{Squamous cell carcinoma antigen (SCC Ag)}

Squamous cell carcinoma antigen (SCC Ag) is the preferred tumor marker for squamous cell cancer. As one of the serine protease family members, SCC Ag is first separated from cervix tumor tissues, and it broadly exists in normal tissues of various organs as well as in malignant epithelial cells. SCC Ag participates in regulating normal and malignant proteolysis, tumor growth in tumor cells, and also assists in diagnosing tumors that originate from squamous epithelial cells. A study by Shufang Qin and another researcher ${ }^{[38]}$ found that the SCC Ag levels of SCLC patients were significantly higher than those in the benign disease group and healthy control group, with a statistically significant difference $(p<0.05)$. In addition to that, with higher clinical staging, the SCC Ag concentrations of SCLC patients were also significantly higher. The difference among the four groups was statistically significant $(p<0.05)$ and the maximum specificity of three combined detection was $100 \%{ }^{[38]}$.

SCC is more valuable to diagnose NSCLC, especially in squamous cell lung cancer, whereas combined detection is more suitable for the diagnosis of SCLC.

\section{D-dimer}

Fibrous protein in blood is activated and hydrolyzed to generate specific degradation products, which are called "fibrin degradation products." D-dimer is considered the simplest fibrin degradation product. Increasing levels of D-dimer indicate secondary hyperfibrinolysis with hypercoagulability. The level of Ddimer is commonly raised in disseminated intravascular coagulation, renal diseases, graft rejection reaction, myocardial infarction, cerebral infarction, pulmonary embolism, venous thrombosis, surgery, tumor disease, infection, or tissue necrosis.

The D-dimer level of cancer patients may also increase, not excluding lung cancer, which has the highest morbidity of malignant tumors. Xiaomin $\mathrm{Bu}$ and other researchers ${ }^{[39]}$ enrolled 300 elderly patients with lung cancer (187 adenocarcinoma, 81 squamous carcinoma, and 32 SCLC), 210 elderly patients with benign lung diseases, and 110 healthy elderly people in their study. The results showed that the D-dimer of the lung cancer group was significantly higher than those in the benign lung diseases group and control group ( $p<0.05$ ); the D-dimer of patients with stage III or IV lung cancer was significantly higher than that of patients with stage I or II lung cancer $(p<0.05)$; the D-dimer of lung cancer patients with distal metastases was higher than those without distal metastasis $(p<0.05)$. D-dimer combined other tumor markers including CYFRA 21-1, NSE, CEA, and CA125 might improve the adjuvant diagnosis effect of lung cancer patients. The high relevance between serum D-dimer concentration and lung cancer staging could be a biological indicator of disease progression, treatment effect, and prognosis of lung cancer.

Meiyu Deng and another researcher ${ }^{[40]}$ found that the D-dimer concentration of lung cancer patients was higher than the healthy control group $(p<0.05)$. In addition, the more advanced the stage, the higher concentration of serum D-dimer in lung cancer patients $(p<0.05)$. There was no significant difference found in the D-dimer levels among lung cancer patients with different pathological patterns $(p>0.05)$. Another study reported that improving the critical value of D-dimer to $981 \mathrm{ng} / \mathrm{ml}$ may effectively evaluate the prognosis of patients with lung cancer ${ }^{[41]}$. Li Gao and another researcher ${ }^{[42]}$ reported that lung cancer patients with advanced staging, poor effect from chemotherapy, and severe disease had high D-dimer levels. Therefore, serum D-dimer test has a certain clinical value in evaluating the chemotherapy effect and the disease progression of lung cancer. 
Since SCLC is the most malignant tumor in lung cancer, its D-dimer can clearly reflect tumor changes. It is expected that more researchers would perform more studies on SCLC, aiding further understanding about the value of D-dimer in diagnosing, staging, and treating lung cancer.

\section{Lactate dehydrogenase (LDH)}

As one of NAD-dependent kinases, lactate dehydrogenase (LDH) is a metalloprotein with zinc ions and one of the important enzyme systems of anaerobic glycolysis as well as gluconeogenesis. LDH can catalyze the related $\alpha$-keto acids as well as facilitate in the reduction-oxidation reaction between propionic acid and L-lactic acid. LDH exists widely in human tissues, with the highest concentration in the kidneys, followed by cardiac muscles and skeletal muscles. The amount of LDH in erythrocytes is about 100 times compared to the normal serum ${ }^{[43]}$. Increased LDH is common in myocardial infarction, liver diseases, hematologic diseases, skeletal muscle injuries, progressive muscular atrophy, pulmonary infarction, and malignant tumors ${ }^{[44]}$.

High LDH concentrations can be detected in many clinical tumor samples. Low LDH can inhibit the growth of tumor cells in vitro and affect tumors in vivo. LDH would not only provide energy to tumor cells, but also prevent injuries to these cells caused by reactive oxygen species (ROS), indirectly promoting tumor cells survival. Furthermore, it can also directly inhibit tumor cell apoptosis; its high expression protects tumor cells from necrosis in anaerobic environment and promote their growth. LDH also plays an important role during tumor invasion and metastases. LDH accelerates lactic acid secretion, regulates metastasis associated proteins, as well as promotes tumor invasion and metastasis by activating epithelial mesenchymal transition (EMT). It also regulates tumor angiogenesis to accelerate the generation of new blood vessels and tumor cell growth. The immune escape of tumor cells is also mediated by LDH by promoting the inhibition of the immune system and its lethal effect ${ }^{[45-47]}$. A study conducted by Fangfang Chen and other researchers ${ }^{[48]}$ showed that the LDH concentrations in SCLC group were higher than those in the NSCLC group, with a statistically significant difference $(p<0.05)$. In addition, the LDH levels of patients with good treatment results were significantly lower compared with the levels in patients with poor treatment results $(p<0.05)$. Through this study, it indicates that serum LDH plays an important role in the diagnosis of SCLC and may be used as an index for evaluating treatment effect. Zhi Wang and other researchers ${ }^{[49]}$ reported that the reduction rate of LDH is positively related to treatment effects $(p<0.01)$, which was exemplified in a lower reduction rate of serum LDH in patients with early recurrence after chemotherapy compared to patients without early recurrence, and there was a statistically significant difference $(p<0.05)$. Zhangchi Pan and other researchers ${ }^{[50]}$ reported that the progression-free survival (PFS) of the low LDH expression group was longer than that of the high LDH expression group among SCLC patients $(5.51 \pm 0.38$ months versus $4.31 \pm 0.28$ months; $p=0.024)$. In the high LDH expression group, PFS was significantly longer with etoposide/cisplatin (EP) regimen compared to that with etoposide/carboplatin (EC) regimen $(4.92 \pm 0.39$ months versus $3.63 \pm 0.38$ months; $p=0.014)$, indicating that LDH is a prediction indicator of treatment effect and is closely related to the treatment effect of SCLC. For newly treated SCLC patients, the level of LDH before treatment has a certain value in evaluating the therapeutic effect and the choice of regimen.

Several studies have demonstrated that LDH levels have value in the diagnosis, staging, effect evaluation, and prognosis of SCLC patients, and the combined detection of other serological indicators may have greater potential.

\section{Neutrophil-to-lymphocyte ratio (NLR)}

In the mid-nineteenth century, Rudolf Virchow had found leukocytes in tumor tissues and pointed out that inflammatory cell infiltration caused by inflammatory reaction surrounding tumor tissues is associated with 
tumor genesis ${ }^{[51]}$. Now, a large number of studies show that inflammatory reaction is indeed associated with tumor genesis and is an important influence factor to prognosis. Massive data present that NLR is closely related to the prognosis in many patients with cancer, including malignant tumors, such as bladder tumor, urinary epithelial carcinoma, NSCLC, esophagus tumor, gastric carcinoma, and pancreatic adenocarcinoma ${ }^{[52-53]}$. Tumor-related inflammatory reaction can exacerbate tumor metastases by inhibiting tumor cell apoptosis and accelerating tumor angiogenesis. However, neutrophils can secrete a large number of vascular epithelial growth factors (VEGFs) to provide a good microenvironment for tumor growth and infiltration ${ }^{[54]}$. As an important medium for the combination of endothelin selectin receptor and endothelin in tumor cells, neutrophils participate in tumor cells metastases ${ }^{[55]}$. Besides, neutrophils also participate in inducing mutation of tumor suppressor genes, the degradation of immunoglobulin, receptor, and complement, as well as promoting proliferation and differentiation of tumor cells.

Serum NLR is an indicator to respond to inflammatory reaction. Generally, high NLR in patients indicates advanced stage, high metastases, and poor prognosis. Xinyue Wang and other researchers ${ }^{[56]}$ retrospective analyzed 235 NSCLC patients with key node, NLR $=2.97$. The results showed that the disease-free survival and overall survival in 1,3, and 5 years as well as the survival after recurrence of 1 or 2 years were higher in the preoperative low NLR group compared to the preoperative high NLR group. A study involving 44 patients with extensive stage SCLC ${ }^{[57]}$ reported that high NLR levels were associated with brain metastases, not receiving chemotherapy, and elevated levels of platelet-to-lymphocyte ratio (PLR), and there was a statistically significant difference $(p<0.05)$; the OS of the high NLR group was lower than that of the low NLR group $(p<0.05)$, indicating that NLR is an independent risk factor influencing the OS of SCLC patients. In another study, Wujun Li and other researchers ${ }^{[58]}$ reported that the recruited SCLC patients with NLR $\geq 3.56$ before chemotherapy had poor prognoses. In addition, the PFS and OS rate in these patients with decreased NLR after chemotherapy were higher $(p<0.01)$.

The relationship between NLR and tumors has gradually gained more interest, where more studies on NLR and the prognosis of malignant tumors have been frequently published. It would be a significant breakthrough in the endeavor to point out a more scientific key node on malignant tumors so that NLR can be confirmed as a prediction indicator of tumors.

\section{Other serological indicators related to SCLC}

It is reported that vascular endothelial growth factor (VEGF), tissue polypeptide specific antigen (TPS), CD56, matrix metalloproteinase (MMP), and tumor abnormal protein (TAP) are associated with the diagnosis, clinical staging, therapeutic effect, and prognosis of SCLC, but they still require further clinical research for confirmation.

\section{Problems and future prospect}

With the continuous development of medical technology, more and more specific tumor markers have been discovered and applicated, providing convenient, efficient, and non-invasive methods for diagnosis and treatment. The combined detection of various tumor markers can improve the detection rate of tumors. In addition, test combined tumor markers and biochemical indicators for SCLC can improve the sensitivity and specificity in detecting SCLC. In these ways, the clinical needs can be met, where patients with SCLC can be recognized earlier, diagnosed, treated, and receive surveillance. The establishment of an optimal combined model has become a priority in regard to SCLC and with continuous advance in precision medicine and gene sequencing techniques, a more precise target localization at molecular diagnostics would become the new area with broad prospects. 


\section{Disclosure statement}

The authors declare that there is no conflict of interest.

\section{References}

[1] Siegel RL, Miller KD, Fedewa SA, et al., 2017, Colorectal Cancer Statistics. CA Cancer J Clin, 67(3): 177-193.

[2] Xu Y, Liu H, Chen J, et al., 2010, Comparisons between the National Comprehensive Cancer Network (NCCN) non-small-cell lung cancer (NSCLC) Clinical Practice Guidelines (Chinese version), the NCCN original edition, and the European Society for Medical Oncology NSCLC Guidelines in 2009. Thorac Cancer, 1(2): 83-86.

[3] Guo Z, Chen X, 2012, Clinical Significance of NSE in the Diagnosis of Small Cell Lung Cancer. Chinese Medical Science, 2(24): 127-128.

[4] Yin Y, Rong C, Zheng H, 2013, Significance of Pro-GRP and NSE in the Differential Diagnosis of Small Cell Lung Cancer. Journal of Radiation Immunology, 26(3): 312-314.

[5] Petrovic M, Bukumiric Z, Zdravkovic V, et al., 2014, The Prognostic Significance of the Circulating Neuroendocrine Markers Chromogranin A, Pro-Gastrin-Releasing Peptide, and Neuron-Specific Enolase in Patients with Small-Cell Lung Cancer. Med Oncol, 31(2): 823.

[6] Zhou J, 2013, Combined Assay of Gastrin-Releasing Peptide Precursor, Tissue Peptide-Specific Antigen and Neuron-Specific Enolase in the Treatment Monitoring of Patients with Clinical Small Cell Lung Cancer. International Journal of Laboratory Medicine, 34(5): 548-550.

[7] Ren LF, Chen MS, Zhang CP, et al., 2009, Clinical Value of Neuron-Specific Enolase for the Diagnosis of Small Cell Lung Cancer. Modern Medical Oncology, 17(12): 2335-2337.

[8] Planchard D, Le Pechoux C, 2011, Small Cell Lung Cancer: New Clinical Recommendations and Current Status of Biomarker Assessment. Eur J Cancer, 47(3): S272-283.

[9] Hurwitz JL, McCoy F, Scullin P, et al., 2009, New Advances in the Second-Line Treatment of Small Cell Lung Cancer. Oncologist, 14(10): 986-994.

[10] Xu X, Tao M, Xie J, 2011, The Predictive Value of CA125 and CA724 on the Efficacy of GP Regimen Chemotherapy in Advanced Lung Cancer. Journal of Clinical Pulmonology, 16(2): 232-233.

[11] Emin EA, Gundyz A, Batum O, et al., 2010, Pre-Treatment and Treatment-Induced Neuron-Specific Enolase in Patients with Small-Cell Lung Cancer: An Open Prospective Study. Arch Bronconeumol, 46(7): 364-369.

[12] Schneider J, Philipp M, Salewski L, et al., 2003, Pro-Gastrin-Releasing Peptide (Pro-GRP) and Neuron Specific Enolase (NSE) in Therapy Control of Patients with Small-Cell Lung Cancer. Clin Lab, 49(12): $35-42$.

[13] Wang WL, Zhou CHY, Zhang GY, et al., 2013, Correlation between Pre-Treatment Lactate Dehydrogenase and Neuronal Enolase Levels and Prognosis of Small Cell Lung Cancer. Chinese Journal of Gerontology, 33(10): 2272-2274.

[14] McDonald TJ, Nilsson G, Vagne M, et al., 1978, A Gastrin Releasing Peptide from the Porcine Nonantral Gastric Tissue. Gut, 19(9): 767.

[15] Miyake Y, Kodama T, Yamaguchi K, 1994, Pro-Gastrin-Releasing Peptide (31-98) is a Specific Tumor Marker in Patients with Small Cell Lung Carcinoma. Cancer Res, 54(8): 2136.

[16] Reeve JR Jr, Cuttitta F, Vigna SR, 1988, Processing of Mammalian Pre-Pro-Gastrin-Releasing Peptide. Ann N Y Acad Sci, 547: 21.

[17] Molina R, Filella X, Augé JM, 2004, Pro-GRP: A New Biomarker for Small Cell Lung Cancer. Clin Biochem, 37(7): 505. 
[18] Pawel JV, Raith H, Holdenrieder S, et al., 2003, Pro-GRP in Serum Enables Diagnosis of Small Cell Lung Cancer. Lung Cancer, 41(2): 201.

[19] Li Y, Fei Z, 2013, Diagnostic Significance of Four Serum Markers in Small Cell Lung Cancer. Shanghai Preventive Medicine, 25(6): 342-344.

[20] Niho S, Nishiwaki Y, Goto K, et al., 2000, Significance of Pro-Gastrin-Releasing Peptide as a Predictor of Relapse of Small Cell Lung Cancer: Comparative Evaluation with Neuron-Specific Enolase and Carcino-Embryonic Antigen. Lung Cancer, 27(3): 159.

[21] Okusaka T, Eguchi K, Kasai T, et al., 1997, Serum Levels of Pro-Gastrin-Releasing Peptide for FollowUp of Patients with Small Cell Lung Cancer. Clin Cancer Res, 3(1): 123.

[22] Shibayama T, Ueoka K, Nishii K, et al., 2001, Complementary Roles of Pro-Gastrin-Releasing Peptide (Pro-GRP) and Neuron Specific Enolase (NSE) in Diagnosis and Prognosis of Small-Cell Lung Cancer (SCLC). Lung Cancer, 32(1): 61-69.

[23] Molina R, Auge JM, Alicarte J, et al., 2004, Pro-Gastrin-Releasing Peptide in Patients with Benign and Malignant Diseases. Tumour Biol, 25(1-2): 56.

[24] Inaji H, Komoike Y, Motomura K, et al., 2000, Demonstration and Diagnostic Significance of ProGastrin-Releasing Peptide in Medullary Thyroid Carcinoma. Oncology, 59(2): 122.

[25] Schauenstein E, Lahousen M, Weblacher M, et al., 1996, Selective Decrease in Serum Immunoglobulin G1. A Tissue Nonspecific Tumor Marker Detecting Early Stages of Gynecologic Malignant Disease with High Efficiency. Cancer, 78(3): 511-516.

[26] Xu S, Huo QE, Zhang M, et al., 2020, The Clinical Diagnostic Value of Combined Serum CA153, CEA, SF, CT and Hcy Tests for Breast Cancer. Journal of Molecular Diagnosis and Therapy, 12(2): 190-194.

[27] Chen XK, Gu CL, Fan JQ, et al., 2020, P-STAT3 and IL-17 in Tumor Tissues Enhances the Prognostic Value of CEA and CA125 in Patients with Lung Adeno Carcinoma. Biomed Pharmacother, 125: 109871.

[28] Luo D, Liu Y, Lin S, et al., 2019, Oncogenic Ras/Squamous Cell Carcinoma Antigen Signaling Pathway Activation Promotes Invasiveness and Lymph Node Metastases in Papillary Thyroid Carcinoma. Oncol Rep, 41(2): 1253-1263.

[29] Yang SW, Li SL, Fang X, 2020, Diagnostic Value of CEA, CA125, NSE, and Pro-GRP for Lung Cancer. Medicine and Health Care, 28(3): 135-137.

[30] Hamesch K, Guldiken N, Mahmoud A, et al., 2020, Serum Keratin 19 (CYFRA21-1) Links Ductular Reaction with Portal Hypertension and Outcome of Various Advanced Liver Diseases. BMC Medicine, 18(1).

[31] Hu YH, Cheng SC, Zhang ZG, et al., 2021, Correlation of CYFRA21-1 Gene Polymorphisms with Clinical Features of Lung Cancer. Journal of Clinical Pulmonology, 26(01): 128-131.

[32] Liu HM, Zhang YX, 2020, Changes in Serum CYFRA21-1 and CEA Levels and Clinical Significance in Patients with Non-Small Cell Lung Cancer at Different Clinical Stages. Journal of Mathematical Medicine, 33(05): 674-675.

[33] Bi H, Fang W, Xue Y, et al., Expression and Clinical Application Value of Four Serological Indicators in Lung Cancer Patients. Anhui Medicine, 41(05): 540-543.

[34] Xiong X, Xiong B, Yao F, 2020, The Value of CYFRA21-1 and BTA Stat Detection in the Diagnosis of Bladder Cancer. Med Info, 33(22): 104-106.

[35] Zhang W, Long SQ, Peng YG, 2020, Clinical Evaluation of Combined Serum CYFRA21-1, HCY and CA15-3 Levels for the Adjuvant Diagnosis of Breast Cancer. Journal of Modern Laboratory Medicine, 35(03): 55-57, 61. 
[36] Zhang D, Li YQ, Gao Y, et al., 2017, Application Value of TPS, CEA, SCC-Ag and CYFRA21-1 in the Assessment of Head and Neck Tumors. Jiangsu Medicine, 43(01): 68-70.

[37] Cui R, 2005, Analysis of CYFRA21-1 and CEA Test Results in Digestive Tract Malignancies. Modern Medicine, (01): 36-37.

[38] Qin SHF, He HN, 2020, Diagnostic Value Analysis of Pro-GRP, SCC-Ag, and CYFRA21-1 for Small Cell Lung Cancer. Journal of Practical Cancer, 35(11): 1814-1816.

[39] Bu XM, Xia L, Li LW, 2020, The Value of D-Dimer Combined with Tumor Markers in the Diagnosis of Lung Cancer in the Elderly. Clinical and Education in Family Medicine, 18(05): 397-400.

[40] Deng M, Zhao J, 2020, Study on the Correlation between Plasma D-Dimer and Lung Cancer Stage, Pathological Type and Prognosis. Journal of Hebei North College (Natural Science Edition), 36(08): 5-7.

[41] Liu Q, Chen Q, Yue CH, 2020, The Value of Increasing the Critical Value of D-Dimer in Assessing the Prognosis of Lung Cancer Patients. Laboratory Medicine, 35(08): 763-766.

[42] Gao L, Liu B, 2020, Clinical Significance of Plasma D-Dimer Assay in the Assessment of Lung Cancer Chemotherapy. Clinical Significance of Plasma D-Dimer Assay in the Evaluation of Chemotherapy for Lung Cancer. Anhui Medicine, 24(06): 1212-1214.

[43] Feng F, Huang WF, 2013, Modern Clinical Diagnostic Test Book, Sichuan Science and Technology Press, 204.

[44] Liu Y, 2017, Practical Laboratory and Clinical, Heilongjiang Science and Technology Press, 72.

[45] Feng Y, Xiong Y, Qiao T, et al., 2018, Lactate Dehydrogenase A: A Key Player in Carcinogenesis and Potential Target in Cancer Therapy. Cancer Med, 7(12): 6124-6136. doi: 10.1002/cam4.1820

[46] Seth P, Csizmadia E, Hedblom A, et al., 2017, Deletion of Lactate Dehydrogenase-A in Myeloid Cells Triggers Antitumor Immunity. Cancer Res, 77(13): 3632-3643. doi: 10.1158/0008-5472.CAN-16-2938

[47] Ding J, Karp JE, Emadi A, 2017, Elevated Lactate Dehydrogenase (LDH) Can Be a Marker of Immune Suppression in Cancer: Interplay Between Hematologic and Solid Neoplastic Clones and Their Microenvironments. Cancer Biomark, 19(4): 353-363. doi: 10.3233/CBM-160336

[48] Chen F, Zhang F, Zhang XQ, et al., 2020, Analysis of the Role of Serum NSE, Pro-GRP and LDH in the Diagnosis and Treatment of Small Cell Lung Cancer. International Infections Diseases (Electronic Edition), 9(01): 47.

[49] Wang Z, Liu YG, Li XF, et al., 2019, Predictive Analysis of Efficacy and Early Recurrence of Small Cell Lung Cancer After First-Line Treatment. Cancer Progress, 17(24): 2948-2951.

[50] Pan Z, Huang X, Zhang L, et al., 2019, Clinical Significance of Serum Lactate Dehydrogenase Measurement in the First-Line Treatment of Small Cell Lung Cancer. Fujian Journal of Medicine, 41(05): 48-51.

[51] Li ZL, 2013, Progress of Research on the Relationship between Neutrophils, Lymphocytes and Neutrophil-Lymphocyte Ratio and Tumors. Medical Review, 19(17): 3128-3131.

[52] Meng Z, Li P, Yan J, et al., 2014, Relationship between Neutrophil and Lymphocyte Ratios and Tumor Characteristics in Patients with Papillary Thyroid Cancer. Anhui Medicine, 18(10): 1880-1883, 1884.

[53] Yang JW, Wu LL, Ni GNT, 2015, Value of Neutrophil-to-Lymphocyte Ratio in the Prognostic Assessment of Radical Cervical Cancer. Journal of Practical Medicine, 31(16): 2662-2665.

[54] Queen MM, Ryan RE, Holzer RG, et al., 2005, Breast Cancer Cells Stimulate Neutrophils to Produce Oncostatin M: Potential Implications for Tumor Progression. Cancer Res, 65(19): 8896-8904.

[55] Mcdonald B, Spicer J, Giannais B, et al., 2009, Systemic Inflammation Increases Cancer Cell Adhesion to Hepatic Sinusoids by Neutrophil Mediated Mechanisms. Int J Cancer, 125(6): 1298-1305.

[56] Wang Z, Liu YG, Li XF, et al., 2014, Predictive Analysis of Efficacy and Early Recurrence of Small Cell Lung Cancer After First-Line Treatment. Cancer Progress, 36(4): 298-302. 
[57] Xu P, Hang C, Liu CY, 2020, Relationship between Peripheral Blood NLR, PLR and Prognosis of Extensive Stage Small Cell Lung Cancer. Traffic Medicine, 34(05): 451-454.

[58] Li W, Chen Z, Zhang B, et al., 2019, Impact of Dynamic Changes in NLR on the Prognosis of SCLC Patients Treated with Chemotherapy. Journal of Practical Oncology, 34(03): 252-256. 